\title{
INDOOR CLIMATE AND ENERGY MODEL CALIBRATION WITH MONITORED DATA OF A NATURALLY VENTILATED DAIRY BARN IN A COLD CLIMATE
}

\author{
Sy Nguyen-Ky,,*, Katariina Penttilä1 \\ ${ }^{1}$ HAMK Tech Research Unit, Häme University of Applied Sciences, Hämeenlinna, Finland. \\ *Correspondence: kysy97@gmail.com, sy.nguyen@hamk.fi
}

\section{HighLightS}

- Indoor climate and energy model of a dairy barn is constructed and calibrated with collected data.

- Long-term monitoring of indoor conditions and electricity consumption greatly facilitates the model calibration process.

- Statistical benchmarks given by guidelines confirm the usability and reliability of the model.

\begin{abstract}
This study demonstrates an application of ICE model calibration by using sensor building metrics in a naturally ventilated dairy house in a cold climate. The barn, at the time of the study, had 70 lactating cows and 30 calves with a total animal area of $1922 \mathrm{~m}^{2}$ and other auxiliary areas of $268 \mathrm{~m}^{2}$. Indoor condition data were collected by four integrated sensors inside the barn for six months, from March to August 2019. IDA ICE 4.8 SP1 simulation software was used to build and simulate the model, with calibration steps conducted first manually, then statistically. Actual weather and indoor condition data during the monitored period were used for calibration; statistical indices of the calibrated model were confirmed by the benchmarks given from ASHRAE Guideline 14-2014, IPMVP version 2016, and FEMP version 4.0 2015. The yielded result was a baseline ICE model, which can be further utilized in the study of energy conservation measures (ECMS), retrofitting feasibility, and ammonia and other contaminant gas emission mitigation. The abovementioned calibration practice and the proposals built on it open a pathway to achieve a higher level of energy efficiency for this type of livestock building.
\end{abstract}

Keywords. Cold weather, Dairy farms, Model calibration, Natural ventilation.

$\mathrm{F}$ inland is the country ranked number one in the world in terms of milk consumption per capita in 2017 , at $457.7 \mathrm{~kg}$ per year. The country's dairy farming was also the largest agricultural sector regarding total turnover. (FAO, 2020) Milk production and production rate depend greatly on various factors, such as feed quality, breeds, farm management, and living conditions of the cows. Over the last two decades, while the number of Finnish dairy farms decreased almost four-fold from the year 2000 and the herd population consequently reduced by $28 \%$, milk production has been significantly increasing, on the contrary (National Resources Institute Finland, n.d.). In addition, the number of large-scale farms (hosting more than 50 cows, in the context of the EU) has been witnessing a linear growth during the period-more than 10 times since the start of the millennium (fig. 1). These trends suggest that more scientific attention should be paid on the construction, maintenance, and monitoring of dairy houses when milk production is more and more centralized and digitalized.

Submitted for review on 25 August 2020 as manuscript number ITSC 14280; approved for publication as a Research Article and as part of the Computer Modeling and Statistics for Agriculture Collection by the Information Technology, Sensors, \& Control Systems Community of ASABE on 2 August 2021.
In 2013, the global livestock industry accounted for $14.5 \%$ of total greenhouse gases (GHG) emission in the world, in which cattle accounts for roughly $65 \%$ of the industry's share, reported by the Food and Agriculture Organization of the United Nations (FAO, 2013). The report also mentioned a potential $30 \%$ cut-down of the GHG emissions by focusing on implementing technologies and advocating modern practices in feeding, animal health and welfare, shelter's energy efficiency enhancement, indoor environment, and manure control systems. However, the situation in Finland since the report was published has not seen positive signs, i.e., the emissions per livestock farm, particularly dairy farms, increased slightly from 9.11 tons $\mathrm{CO} 2 \mathrm{e}$ per dairy cow in 2000 to 10.53 tons CO2e per dairy cow in 2018. The rise was explained by the increases from the expanded land use for fodder area, feed-induced emissions (manure and enteric fermentation), and the energy used (heating and electricity) (National Resources Institute Finland, 2020). These numbers pose questions on how energy efficient a dairy barn can get, by which measures, and how it will contribute to the GHG emission mitigation progress. Research on this topic is even more meaningful in the context of an increasing number of large-scale dairy farms predictably in years to come. 
Herd size vs milk production in

Finland in $2000-2019$

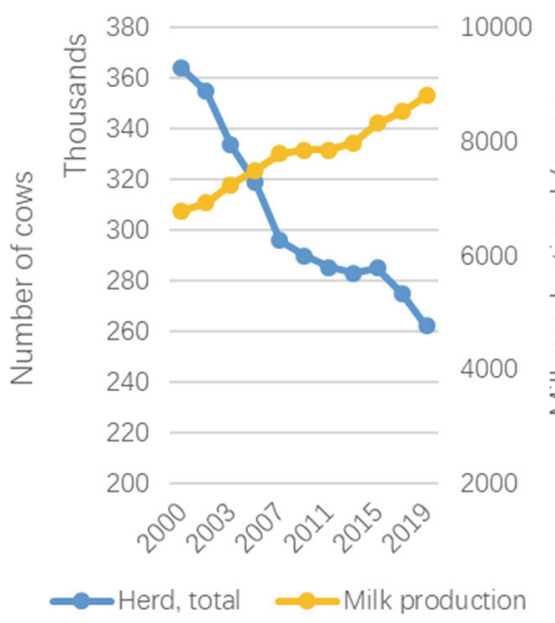

Number of farms in Finland

in $2000-2019$

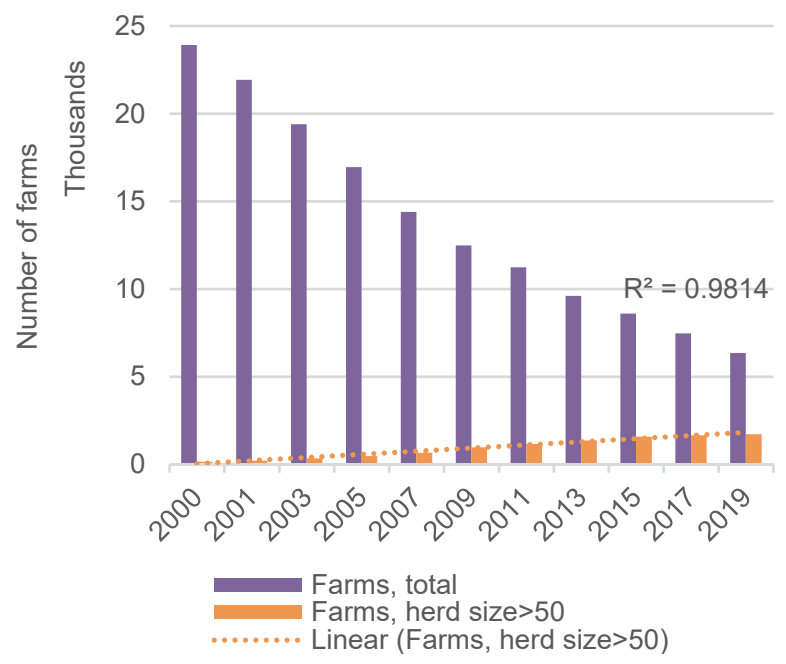

Figure 1. Overview of the Finnish dairy industry during the 2000-2019 period. (Data retrieved from (National Resources Institute Finland, n.d.).

Milk production depends on many factors, as mentioned above, in which indoor environment and feeds are the two most critical ones. In Finland, although the subarctic climate does not cause a serious challenge to the heat sensitivity of the dairy cows, it is the cold and prolonged winter that hinders the quality and stable maintenance of a good living environment, i.e., in-range temperature, relative humidity, and sufficient fresh air change. Most dairy barns in Finland are naturally ventilated with large side and ridge openings. This type of ventilation thus depends directly on the outdoor climate conditions, which means the ventilation rate is controlled mainly through the opening of side and ridge windows. Ventilation rate in naturally ventilated buildings has a considerable impact on the indoor environment of dairy barns; especially in cold climate regions, its control is crucial in order to cut off the extremes in wintertime and mitigate the effects of physiologic heat stress to the cows in the summertime. Previous research works regarding naturally ventilated dairy barns, particularly the ventilation, were conducted by different methods. Huang and Guo (2018) estimated the ventilation rate in a naturally ventilated barn in Canada by a simplified $\mathrm{CO}_{2}$ mass balance method, where the ventilation rate equation is explicitly enhanced by an empirical correction factor. This equation was adopted from the paper of Pedersen and Sällvik (2002) and was also applied in the study of Ngwabie et al. (2011) to measure the emission factors in a dairy barn in Sweden. In Shen et al. (2013) exclusive study about the control of natural ventilation in barn building, the ventilation rate was calculated by using computational fluid dynamics (CFD) tools, which required more intensive computing power and time. In return, the result of this method was of higher precision and reliability. The calculation method for the ventilation rate is crucial in studying the variation of other GHG emissions and ammonia concentration inside the dairy barns. Therefore, the method selection depends heavily on the expected level of accuracy of the result and the available data. In principle, the method is chosen such that the amount of assumed information is minimal, and most importantly, to keep the result as objective as possible.

Buildings nowadays are carefully designed and planned before they are built. Many countries even require an energy performance calculation before they grant the building permit. ICE model can already be built and simulated at this preliminary stage to estimate the consumption of energy and indoor conditions, which is required in the building permit application. However, the gap between the predicted performance in the design and construction stage and the observed one during the operation stage has been reported to be huge in many cases (Gucyeter, 2018). This has pushed forward a need for more reliable methodologies in increasing the building energy efficiency level and its functional operation in the post-occupancy stage using computational simulation tools. Building energy simulation was then introduced as a bright, new direction to solve this problem in both the assessment of the current level of performance and the energy retrofitting feasibility study of the buildings. However, the complex and extensive calculation of the energy performance of building without the proper quality of input data may cause a low confidence level in the simulated results. If that inadequate model were to be employed in further study, the discrepancies might have gone larger and larger. Therefore, the task of choosing, assuming, filtering to get the as-close-aspossible input data and minimizing the errors in simulation has drawn more attention, so-called the calibration process. Calibration has been widely in practice and considered the essential step in ECM project. In order to achieve reliable results when evaluating any ECM, one must produce a highquality building energy model (BEM), i.e., calibrated BEM. Also, calibration can be applied in the commissioning services of existing buildings, or, in fault detection and diagnosis (FDD) activities. However, many modelers have reported that this process might bring critical issues to the practice. Bandera and Ruiz (2017) conducted a thorough literature review and pointed out that the hindrances are: 
- the great amount of input information and measured data over 12 months (as recommended in ASHRAE Guideline 14-2014),

- its time- and manpower-consuming,

- modeler's skills and experience, and

- no universal standardized approach.

In this article, we use the IDA Indoor Climate and Energy simulation tool version 4.8 SP 2 from EQUA Simulation AB as our working tool. IDA ICE uses the mass-pressure balance equation method to calculate the natural ventilation rate, with a choice to either include a multi-node vertical 1D temperature gradient model (in the detailed zone model) or assume the air as well-mixed (in the simplified zone model). In the simplified zone model, the pressure rise due to buoyancy or wind (data from the climate file) is modelled and pressure resistances through leaks and openings are calculated from the input geometry of the barn building. Based on the obtained mass flow and the balance equations for $\mathrm{CO}_{2}$ concentration, humidity, air mass, energy, all the variables are also obtained, simultaneously. Using this software allows the modelling work to be more flexible than those two above-mentioned methods - the simplified $\mathrm{CO}_{2}$ mass balance method with a correction factor, and the CFD simulation method: one has a lower level of detail and the other one is too complex, time- and power-consuming, and expensive for longer simulation period. The details of the mathematical models mentioned above can be read from these two references (Bring, Sahlin, \& Vuolle, 1999; EQUA Simulation $\mathrm{AB}, 2013)$. IDA ICE also handles the overall energy perspectives of the barn-space heating, domestic hot water heating, and electricity for machinery and milk cooler. Furthermore, with the widely available IoT sensor equipment nowadays, most indoor condition indicator data can be monitored and collected effortlessly. For these reasons, IDA ICE is a promising tool to efficiently practice building indoor climate modelling, calibrating, and validating.

Given the background information, the main objective of this research paper is to present the complete process of constructing an ICE baseline model of a dairy barn and the model calibration procedure with monitored data.

\section{Materials ANd Methods DESCRIPTION OF THE DAIRY BARN}

The free-stall dairy barn Mustiala (in Finnish: Mustialan Navetta) is located at Mustialantie 105, 31310 Mustiala, Finland $\left(60^{\circ} 52^{\prime} 55.0^{\prime \prime} \mathrm{N}, 2^{\circ} 14^{\prime} 53.9^{\prime \prime} \mathrm{E}\right)$. The location is in southern Finland with a typical subarctic climate-prolonged freezing winter with modest sunlight. At the time the measurements were collected, the barn was hosting 100 cows (70 lactating cows and 30 heifers) of the maximum capacity of 193 cow places (90 lactating cows, 71 heifers, and 32 calves). The building has a total floor area of $2245 \mathrm{~m}^{2}$, of which the animal area remains $1922 \mathrm{~m}^{2}$ and the rest is for functional auxiliary spaces, i.e., offices, cold rooms, feed storages, and technical rooms. The animal area is divided into various sub-areas according to the age and stage category of the animals (dry, calving cows, heifers, calves), the milking robot station, and the feed room (fig. 2). There are four pen rows with slatted floor stretching lengthwise of the building, but not

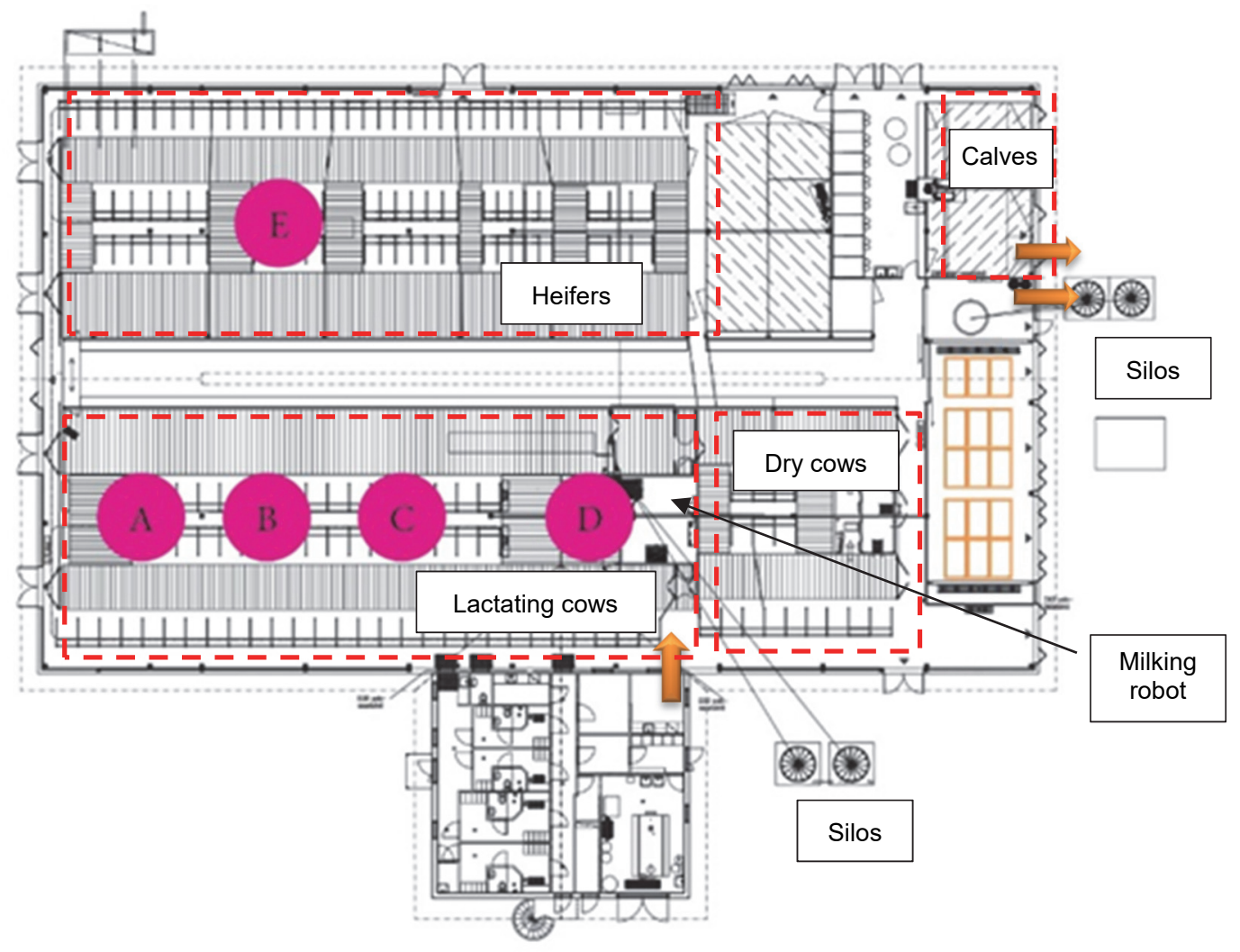

Figure 2. A floor plan view of the barn complex and the locations of the indoor condition sensors in the animal area, marked with letters A-E (adapted from Koskela et al., 2020). 
tail-to-tail, under which the gravity flow manure channels run. The depth of the manure channel is 1.1 to $1.3 \mathrm{~m}$ and is connected to form a system of manure collecting channel under the barn. There is an intermediate manure storage pit at the northwest corner of the building, from which the slurry manure will be pumped to the two outdoor manure pits. The scraping of manure on the slatted floor is scheduled twice a day. The barn uses robot milkers, which explains why there is no milking parlor as in traditional barns.

The office part was designed and constructed according to the building codes for the office building category. However, the animal area was thinly insulated with a significant window to total surface area ratio - a typical design of curtain wall window for naturally ventilated livestock building in Finland (fig. 3). The opening of these windows was manually controlled based on the barn keepers' experience and weather condition observations. Besides, there was a fan moving the exhaust air from the office building block to the animal area and two exhaust fans on the outer wall of the animal area. The fans are marked with orange arrows in figure 2. The exhaust air fan from the office block to the animal area has a maximum design flow of $150 \mathrm{~L} / \mathrm{s}$, which is insignificant in comparison to the total volume of the animal area. One of the other two fans is placed in the calf room to cool the calves in case of hot summer and the other one is in the feed room to ventilate the feed in the late spring - early summer and autumn. These two fans did not involve in the ventilation of the animal area at all during the monitored period. Therefore, the effect of these two exhaust fans was not considered in the model calibration. The heating in the animal area is mainly due to the heat emitted from the cows. More particularly, the calf room has heat radiators, which have a maximum heating power of $4.5 \mathrm{~kW}$. These are trivial in terms of providing heat for the hall and are only meant to locally warm up the newly born calves, if any. The heating for the office block was designed normally as for office, which is not of the focus in this study. Thus, the animal area can be considered as naturally ventilated and was modelled as a single zone.

\section{Measurements and data Collection}

The indoor condition data collection period for this study lasted six months, from 1 March 2019 to 31 August 2019.
The data covered the transitional period from winter to spring and the whole summer of 2019. Data readings were collected and sent to the database every 15 min through LoRaWANTM, which is a cloud-based medium access control (MAC) layer protocol, from a set of four ELSYS ERS-CO $\mathrm{CO}_{2}$ sensors located at the middle lines as depicted (A-D) in figure 2. Three sensors (A-C) were installed at the height of $1.6 \mathrm{~m}$ from the floor at the lactating cow category space, and the fourth one (D) was attached to the milking robot. Two other ELSYS ELS-2 sensors at E were also installed for vertical temperature, air pressure, and relative humidity gradient impact analysis, which were not used in this study. Five indoor conditions were observed through the sensor set, i.e. air temperature, relative humidity, lighting level, $\mathrm{CO}_{2}$ concentration, and motion level (Koskela et al., 2020). Due to the heat dispersed locally from the robot, sensor D was not considered in the analysis because the local conditions were influenced by the robot milking station. Therefore, hourly average values were calculated for each measurement only from sensors A-C to compare to the simulated results later when calibrating and validating the model. During July and August, there was a fault in the readings of the RH in sensor $\mathrm{B}$ and $\mathrm{C}$; hence, the reliable and usable $\mathrm{RH}$ data is taken from March until the end of June only. Observed weather data of the corresponding period was retrieved from the Finnish Meteorology Institute open data, at the Jokioinen meteorological station, which is located approximately $16 \mathrm{~km}$ to the west of the barn. Instantaneous weather values observed at a 10-min interval were converted to hourly average following the format of a weather file used in IDA ICE. The author wrote a customized script in $\mathrm{R}$ (version 3.6.1) to process the raw collected data and produce the observed weather file for the simulation software. For electricity calibration, the consumptions from 28 December 2018 to 12 March 2019 and from 13 March 2019 to 12 July 2019 were used. Electricity used inside the barn and the office building includes lighting, equipment, machinery, milking robots, floor scraping robots, pumping system, and the cooling room load.

\section{WORKING TOOLS}

As mentioned above, the dairy barn was modelled and simulated in IDA ICE simulation software. IDA ICE is a simulation program from EQUA Simulation $\mathrm{AB}$. It is a

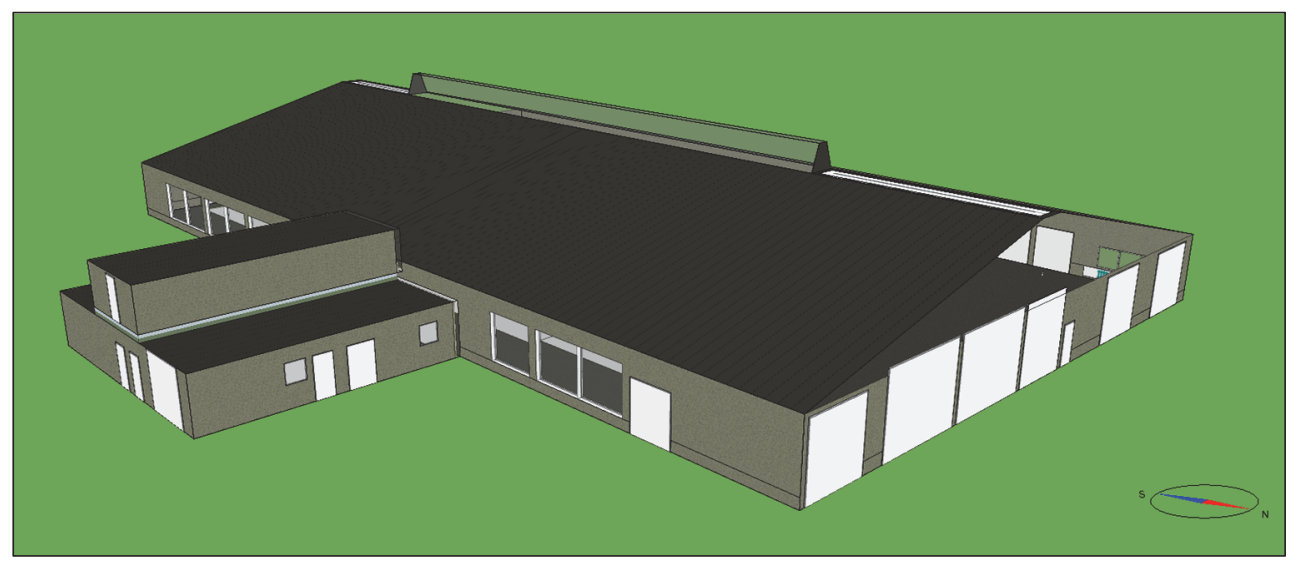

Figure 3. The 3D model of the barn complex in IDA ICE. 
popular whole building simulation tool in the Nordic and Baltic regions because of its origin and its good adaptability to the building codes as well as the local languages. The advantage of using IDA ICE is that it allows the modeler to customize their models in both general and advanced modes, i.e., transparent mathematical model enables the inspections of a great number of parameters and algorithms underlaid. This dynamic simulation software IDA ICE has been validated and certified with standardized tests, such as ASHRAE 14-2014, CEN Standard EN 13791, CEN Standard EN 15255- and 15265-2007, and IEA SHC Task 34 (EQUA Simulation AB, n.d.). It was employed in many recent studies, proving its strong reliability and robustness, as in Häkämies et al. (2015) and Simson et al. (2019). Along with the simulation program, a script developed in $\mathrm{R}$ were also developed in the calibration processes for statistical index calculation and result visualization.

\section{ICE Model Calibration}

Building energy model calibration, as known as tuning the input parameters to fit the simulated results to the real monitored measurements. A baseline model is a calibrated model of the building, which behaviorally reflects the thermal characteristics of that building. Officially, ASHRAE Guideline 14-2014 defines "calibration" as the "process of reducing the uncertainty of a model by comparing the predicted output of the model under a specific set of conditions to the actual measured data for the same set of conditions" (ASHRAE, 2014).

A combination of several calibration methods can be employed as long as they fit the end purposes of each specific case in the end. In this study, the model calibration was firstly done by iteratively manual fit-the-graph technique, then, by the confirmation of statistical indices. The schematic process of the manual iterative calibration was described through a flowchart by Gucyeter (2018). The energy/electricity consumption and the indoor air temperature of the simulated zone, in some cases, are usually used for calibration. The two official statistical indices that the final calibration step followed were the coefficient of variation of the root-mean-square error [CV (RMSE)] and the normalized mean bias error (NMBE). Besides, the mean absolute percentage error (MAPE) was also used. The equation of the indices are presented from (1) to (3). The values of these indices produced by the calibrated model will confirm the usability of the end model.

$$
\begin{aligned}
\mathrm{CV}(\mathrm{RMSE}) & =\frac{1}{\bar{y}} \times \sqrt{\frac{\sum_{i}^{n}\left(y_{i}-\hat{y}_{i}\right)^{2}}{(n-1)}} \\
\mathrm{NMBE} & =\frac{\sum_{\mathrm{i}}^{\mathrm{n}}\left(\mathrm{y}_{\mathrm{i}}-\hat{\mathrm{y}}_{\mathrm{i}}\right)^{2}}{(\mathrm{n}-1) \times \overline{\mathrm{y}}} \\
\mathrm{MAPE} & =\sum_{\mathrm{i}}^{\mathrm{n}}\left|\frac{\mathrm{y}_{\mathrm{i}}-\hat{\mathrm{y}}_{\mathrm{i}}}{y_{i}}\right|
\end{aligned}
$$

where:

$\mathrm{y}_{\mathrm{i}}=$ measured value at instance $\mathrm{i}$

$\hat{\mathrm{y}}_{\mathrm{i}}=$ simulated value at instance $\mathrm{i}$

$\mathrm{n}=$ number of data points

$\overline{\mathrm{y}}=$ mean of the measured values.

The ASHRAE Guideline 14-2014 (ASHRAE, 2014) provides the recommended benchmarks for the NMBE and $\mathrm{CV}(\mathrm{RMSE})$ as the criteria for an hourly calibrated model, which are shown in table 1 . Hourly simulations and calibration enhance the model reliability if followed by studies with high-accuracy requirements, e.g., in energy consumption prediction, automation controls, fault diagnostics, etc. Hourly calibration requires more effort and time for the calibration process; not to mention, hourly simulation also consumes more computing power. The latest versions of several other popular M\&V guidelines, for instance, the International Performance Measurements and Verification Protocol (IPMVP) version 2016 (Efficiency Valuation Organization, 2016) and the Federal Energy Management Program (FEMP) version 4.02015 (Federal Energy Management Program, 2015), also refer to the values for statistical analysis of simulation errors by ASHRAE Guidelines 14. Regarding the MAPE index, there is not yet any official benchmark range established from the concerned guidelines. However, as usually used in calibration practice, we will consider a MAPE below $10 \%$ as an acceptable value.

In this work, the initial BEM was constructed based on the information from the as-built structural, architectural, and HVAC drawings of the dairy barn. Site orientation, wind profile, and shading objects from the surroundings were also added accordingly. The animal area was modelled as one single thermal zone, albeit its large share of floor area and volume. The hall is also the main body of focus in this study. Occupation schedules, machinery, lighting, etc., were also considered at this stage. This is to ensure that the BEM can effortlessly reach the first level of calibration, as described in the work of Mustafaraj et al. (2014). On the same topic, Fabrizio and Monetti (2015) did an extensive literature review and produced a list of the influencing parameters to handle. Based on this list and the elimination method through the modeler's experience, the most uncertain factors which can affect the reliability of the calibration process in this work were made italic in table 2. Alternatively, this step could also be studied more closely by conducting the sensitivity analysis (SA), which was done in many previous research works such as in (Calleja Rodríguez et al., 2013; Serbouti et al., 2018). SA, usually conducted in parallel with uncertainty analysis (UA), is highly useful when the model is complicated, or when there is an insufficient amount of input information. SA and UA act as a decision-making supportive tool, especially in building design stage or retrofitting study. In this dairy barn case, SA and UA will be performed in the HVAC and energy system improvement

Table 1. Benchmarking values for ICE model calibration.

\begin{tabular}{cccc}
\hline & \multicolumn{3}{c}{ Index } \\
\cline { 2 - 4 } $\begin{array}{c}\text { Hourly Calibration } \\
\text { Benchmarked by }\end{array}$ & NMBE & CV (RMSE) & MAPE \\
$(\%)$ & $(\%)$ & - \\
\hline ASHRAE (14-2014) & \pm 10 & 30 & 10 \\
\hline- & & & \\
\hline
\end{tabular}


Table 2. Uncertainty sources in building calibration and the most influential parameters in this work in italic (adapted from Heo, 2011).

\begin{tabular}{|c|c|c|}
\hline Uncertainty Category & General Factors & Particularly in this Case Study \\
\hline Scenario & $\begin{array}{l}\text { Outdoor weather conditions } \\
\text { Building occupancy schedule }\end{array}$ & - \\
\hline Building physics/operation & $\begin{array}{c}\text { Building envelope characteristics } \\
\text { Internal gains } \\
\text { HVAC systems } \\
\text { Operation and control settings }\end{array}$ & $\begin{array}{l}\text { Share of heat and moisture load } \\
\text { from cow and machinery } \\
\text { Curtain wall manual opening schedule }\end{array}$ \\
\hline Model inadequacy & $\begin{array}{l}\text { Modelling assumptions } \\
\text { Simplification in the model algorithm } \\
\text { Ignored phenomena in the algorithm }\end{array}$ & $\begin{array}{l}\text { All lactating, transition, dry cows are modelled } \\
\text { as one group; one calf equals half a cow } \\
\mathrm{CO}_{2} \text { source is only from animals, } \\
\text { not including from manure or feed } \\
\text { The air in the animal area is well-mixed, } \\
\text { no temperature gradient was considered }\end{array}$ \\
\hline
\end{tabular}

study; however, they are not performed in the scope of this article.

Then, the first calibration attempts were conducted. At this stage, non-routine adjustments were also considered. Iterative calibration runs had been performed until a satisfying level was achieved visibly by graphical comparison, before using the statistical indices to finally confirm its usability. The initial and calibrated values are shown in table 3 . Four outputs from the model were used to calculate the calibration indices: temperature, $\mathrm{RH}, \mathrm{CO}_{2}$ concentration, and electricity consumption. The aim of the work is to construct a model which produces in-range statistical indices for all four so that the model can be declared as a "calibrated baseline model" in terms of indoor climate and energy. Since the opening of the curtain wall window was irregular with manual adjustment and mostly based on outdoor temperature, it was generalized as an outdoor air temperature dependent segmented curve. The ratio of window opening area to the total area of the window is zero, or completely closed, at $-30^{\circ} \mathrm{C}$. In the case of the curtain wall, this ratio will never get the value 1 , which means completely open. The curve is divided into three segments by three mobile points and one fixed point at $-30^{\circ} \mathrm{C}$. The GenOpt generic optimization software will sample and run the simulations until it achieves the minimum value for our objective function. The GenOpt generic optimization software is incorporated for direct use from the interface of IDA ICE software. The objective function for the curve in the optimization-based calibration step was established to minimize the difference between the simulated and measured indoor air temperature of the animal area during the monitored period. GenOpt will return the coordinates of the three points which define the three segments.

\section{RESULTS AND DISCUSSION \\ Model Calibration Results \\ Temperature, Relative Humidity, and Carbon Dioxide Concentration Calibration}

Figure 4 depicts the calibration result for each source, the measured versus simulated and the two calculated indices. Graphically, the measured and simulated temperature displays similar trends during the studied period. There was a slight mismatch in late March to the start of April when the measure indoor temperature peaked and plummeted, while the simulated temperature was quite stable. Regarding $\mathrm{CO}_{2}$ concentration, there is a wider diurnal fluctuation in the actual data while the simulated values perform more stably. This pattern can also be seen when observing the calibration result of RH, but in this case, the actual values are fluctuating more than the simulated ones. Nonetheless, both the NMBE and CV(RMSE) indices of all three sources fall within the acceptable ranges provided by all three guidelines presented in table 1. Based on that, the baseline model can be confirmed as "calibrated" in terms of indoor conditions.

Table 3. Initial and final values of input variables during the calibration process.

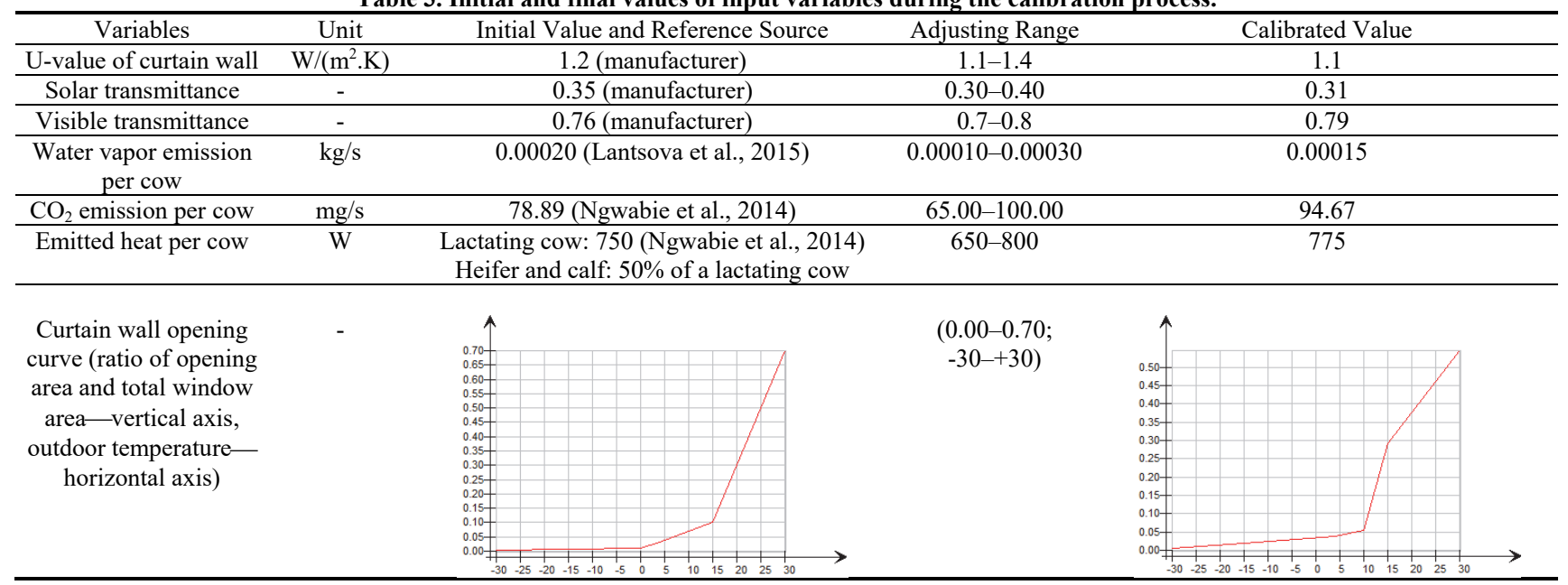


CO2 concentration comparison between simulated and actual data

calibrated model, 6 months, 1 March - 31 August, 2019

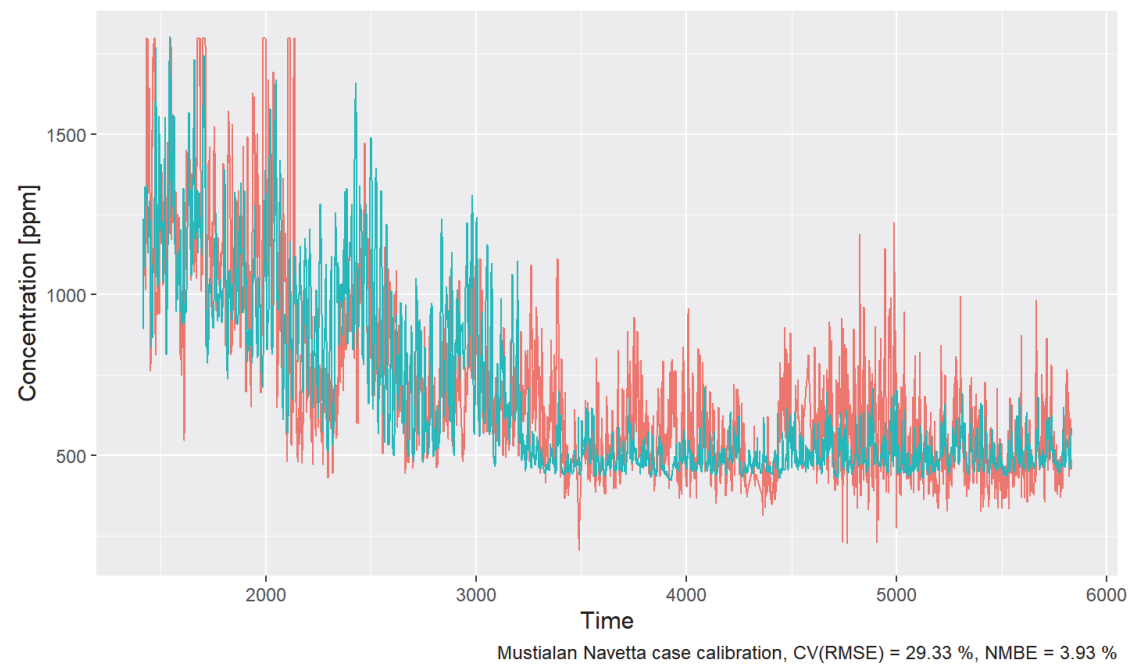

Temperature comparison between simulated and actual data calibrated model, 6 months, 1 March - 31 August, 2019

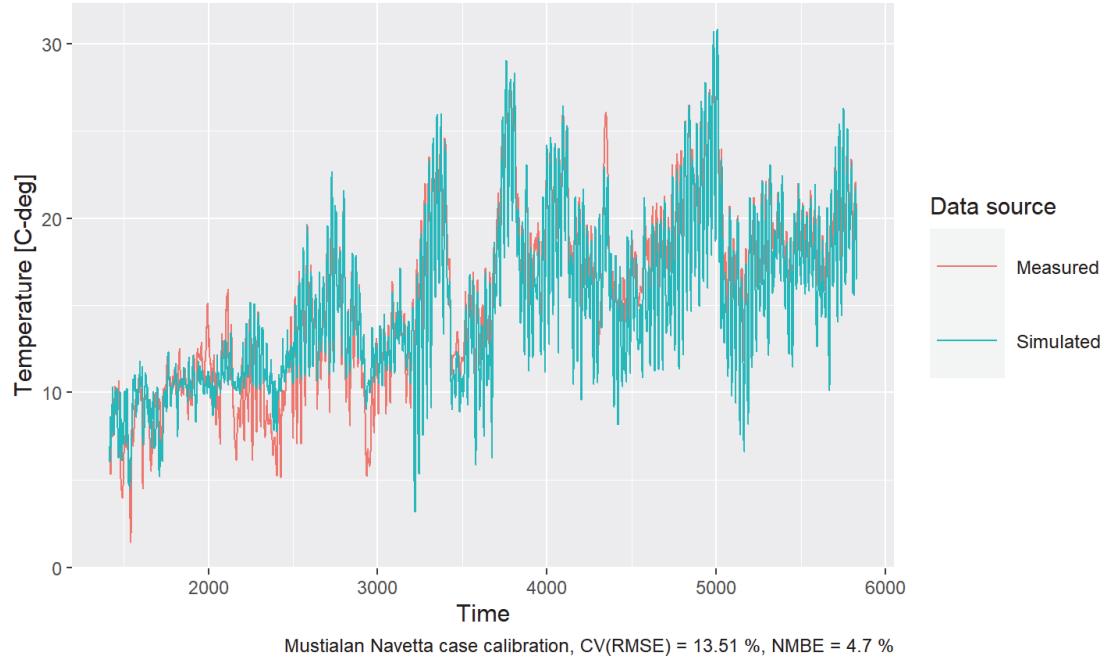

Relative humidity comparison between simulated and actual data calibrated model, 4 months, 1 March - 31 June, 2019

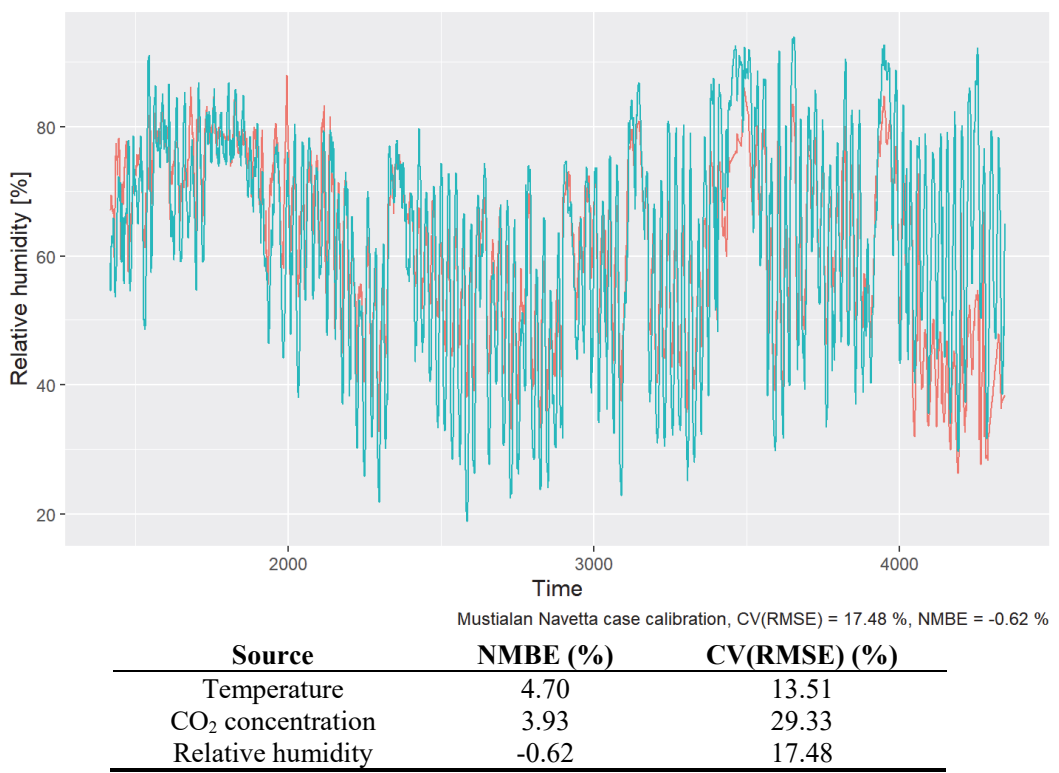

Figure 4. Indoor condition calibration results. 


\section{Energy Calibration}

The measured versus the simulated electricity consumptions and the MAPE index are showed in table 4. Usually, this statistical index is not recommended to be used alone because it can cancel out the negative and positive errors. However, the barn electricity load, unlike in other types of buildings, operates stably and regularly as long as the number of cows in the barn remains unchanged, i.e., all the chores will be running as scheduled. In other words, there is no significant difference among the electricity readings in different months or different times of the year. The electricity load calibration with MAPE index is about $1 \%$, which is well below the said acceptable threshold $10 \%$. Therefore, the electricity consumption from the model is considered calibrated.

\section{Baseline Model Applications}

The production of a calibrated baseline model laid the first stone in developing a digital copy of the physical performance of the actual dairy barn, especially the indoor environment. Studies on GHG emission mitigation, optimal ventilation control inside the dairy barn require a reliable starting point of the physical behavior of the building. Furthermore, with such a conventional calibration methodology as presented, the evaluation of multiple ECMs can be observed separately and the final joint impact can be assessed more accurately (Federal Energy Management Program, 2015). Although the approach based on long-term monitoring of energy and indoor conditions took time and resources, the baseline model opens a wider pathway to further meticulous retrofitting options in the future. One example is that the optimal control of the side window opening areas in case a retrofitting of an automatic control system is installed. The modeler can also implement and tune a control scheme that gives the output signal for the window opening concerning the effect of indoor air temperature, $\mathrm{RH}$, and $\mathrm{CO}_{2}$ concentration. The air exchange rate together with the $\mathrm{CO}_{2}$ concentration level is a crucial factor in the calculation of the ammonia emission and other contaminant gases inside the dairy barns and other cattle buildings. Another application of the baseline model in this case study is to test the feasibility of installing an exhaust air heat pump (EAHP) in the heating system of the office part of the barn. The temperature inside the barn hall is generally above $5^{\circ} \mathrm{C}$ even on the coldest day of winter, which is ideal for a highly efficient EAHP to operate, making use of the heat produced by the cows. The sizing of the heat pump and assessment of the potential savings from it can also be simulated and calculated to support the decision-making process. Additionally, it is of high importance for livestock buildings to anticipate the changes in their operation versus its physical behavior concerning the constantly changing number of animal heads. This model is capable of conducting such task smoothly, or so-called functional scalability.

Table 4. Electricity consumption calibration result.

\begin{tabular}{cccc}
\hline $\begin{array}{c}\text { Electricity } \\
\text { Consumption }\end{array}$ & $\begin{array}{c}\text { 27 Dec. 2018- } \\
12 \text { March 2019 }\end{array}$ & $\begin{array}{c}\text { 13 March 2019 - } \\
\text { 12 July 2019 }\end{array}$ & \multirow{2}{*}{ MAPE } \\
\hline Measured & $40525 \mathrm{kWh}$ & $44740 \mathrm{kWh}$ & \multirow{2}{*}{$1.04 \%$} \\
\hline Simulated & $40352 \mathrm{kWh}$ & $44464 \mathrm{kWh}$ & \\
\hline
\end{tabular}

\section{CONCLUSIONS}

The ICE model of our naturally ventilated dairy barn in a cold climate attained a successful calibration process. The long-term monitoring of indoor conditions and energy consumption facilitated a reliable model calibration. This work proves that a satisfied baseline model of a barn building can be achieved by using a combination of calibration methodologies with the confirmation of statistical indices given by the three most popular guidelines in the field of measurement and verification, ASHRAE 14, IPMVP, and FEMP.

Calibrated baseline model eases the impact evaluation and savings potential of ECMs, delivers a tool to study the feasibility to enhance the ICE control systems, and most importantly, supports the decision-making process in the efficient management of livestock buildings. The calibrated model is also characterized by the scalability, i.e., it enables the barn managers to foresee the possible scenarios in case the herd size changes so that the corresponding measures can be promptly taken. The construction and calibration of such an ICE model of this type of building are significant when the improvement of energy efficiency, as well as mitigation of the GHG in the livestock industry, is attracting more attention in the future.

\section{REFERENCES}

ASHRAE. (2014). Measurement of Energy and Demand Savings. In ASHRAE Guideline 1 4-2014 (Vol. 8400). Atlanta, GA.

Bandera, C. F., \& Ruiz, G. R. (2017). Towards a new generation of building envelope calibration. Energies, 10(12). https://doi.org/10.3390/en10122102

Bring, A., Sahlin, P., \& Vuolle, M. (1999). Models for building indoor climate and energy simulation, A Report of Task 22 Building Energy Analysis Tools. Report of IEA SHC Task, (December). Retrieved from https://www.ieashc.org/Data/Sites/1/publications/t22brep.pdf

Calleja Rodríguez, G., Carrillo Andrés, A., Domínguez Muñoz, F., Cejudo López, J. M., \& Zhang, Y. (2013). Uncertainties and sensitivity analysis in building energy simulation using macroparameters. Energy and Buildings, 67, 79-87. https://doi.org/10.1016/j.enbuild.2013.08.009

Efficiency Valuation Organization. (2016). IPMVP Core Concepts 2016. (October). Retrieved from https://evoworld.org/en/products-services-mainmenuen/protocols/ipmvp

EQUA Simulation AB. (n.d.). Validation \& certifications Simulation Software.

EQUA Simulation AB. (2013). User Manual IDA Indoor Climate and Energy Version 4.5. Retrieved from http://www.equaonline.com/iceuser/pdf/ice45eng.pdf

Fabrizio, E., \& Monetti, V. (2015). Methodologies and advancements in the calibration of building energy models. Energies, 8(4), 2548-2574. https://doi.org/10.3390/en8042548

FAO. (2013). Tackling climate change through livestock - A global assessment of emissions and mitigation opportunities, Food and Agriculture Organization of the United Nations.

FAO. (2020). FAOSTAT - Food and agriculture data.

Federal Energy Management Program. (2015). M\&V guidelines: measurement and verification for performance-based contracts -Version 4.0. U.S. Department of Energy, 3(November), 1-108. 
Gucyeter, B. (2018). Calibration of a building energy performance simulation model via monitoring data. ASHRAE and IBPSA-USA Building Simulation Conference, 542-549.

Häkämies, S., Hirvonen, J., Jokisalo, J., Knuuti, A., Kosonen, R., Niemelä, T., ... Pulakka, S. (2015). Heat pumps in energy and cost efficient nearly zero energy buildings in Finland.

Heo, Y. (2011). Bayesian Calibration of Building Energy Models for Energy Retrofit Decision-Making Under Uncertainty. 1116.

Huang, D., \& Guo, H. (2018). Diurnal and seasonal variations of greenhouse gas emissions from a naturally ventilated dairy barn in a cold region. Atmospheric Environment, 172, 74-82. https://doi.org/10.1016/J.ATMOSENV.2017.10.051

Koskela, O., Virtanen, K., Kukkamäki, J., Aronen, I., \& Kunttu, I. (2020). Navetta 4.0 - Navetan olosuhteiden jatkuva mittaaminen. https://doi.org/10.13140/RG.2.2.29679.18086

Lantsova, E., Vtoryi, V., \& Vtoryi, S. (2015). Investigation of water evaporation from cattle manure. Engineering for Rural Development, 14(January), 590-593.

Mustafaraj, G., Marini, D., Costa, A., \& Keane, M. (2014). Model calibration for building energy efficiency simulation. Applied Energy, 130, 72-85. https://doi.org/10.1016/j.apenergy.2014.05.019

National Resources Institute Finland. (n.d.). Statistics of the National Resources Institute Finland.

National Resources Institute Finland. (2020). Milk production National Resources Institute Finland.

Ngwabie, N. M., Jeppsson, K. H., Gustafsson, G., \& Nimmermark, S. (2011). Effects of animal activity and air temperature on methane and ammonia emissions from a naturally ventilated building for dairy cows. Atmospheric Environment, 45(37), 6760-6768.

https://doi.org/10.1016/j.atmosenv.2011.08.027

Ngwabie, Ngwa M., Vanderzaag, A., Jayasundara, S., \& Wagner-Riddle, C. (2014). Measurements of emission factors from a naturally ventilated commercial barn for dairy cows in a cold climate. Biosystems Engineering, 127, 103114. https://doi.org/10.1016/J.BIOSYSTEMSENG.2014.08.016

Pedersen, S. \&, \& Sällvik, K. (2002). International Commission of Agricultural Engineering, Section II 4th Report of Working Group Climatization of Animal Houses Heat and moisture production at animal and house levels.

Serbouti, A., Rattal, M., Boulal, A., Harmouchi, M., \& Mouhsen, A. (2018). Application of sensitivity analysis and genopt to optimize the energy performance of a building in Morocco. Intl. J. of En. and Tech.(UAE), 7(4), 2068-2074. https://doi.org/10.14419/ijet.v7i4.13280
Shen, X., Zhang, G., Wu, W., \& Bjerg, B. (2013). Model-based control of natural ventilation in dairy buildings. Computers and Electronics in Agriculture, 94, 47-57. https://doi.org/10.1016/j.compag.2013.02.007

Simson, R., Arumägi, E., Kuusk, K., \& Kurnitski, J. (2019). Redefining cost-optimal nZEB levels for new residential buildings. E3S Web of Conferences, 111, 03035. https://doi.org/10.1051/e3sconf/201911103035

\section{NOMENCLATURE}

ASHRAE $=$ The American Society of Heating, Refrigerating and Air-Conditioning Engineers

BEM = Building Energy Model

BIM = Building Information Model

$\mathrm{CEN}=$ European Committee for Standardization

CFD = Computational Fluid Dynamics

$\mathrm{CO} 2 \mathrm{e}=$ carbon dioxide equivalent

$\mathrm{CV}(\mathrm{RMSE})=$ Coefficient of Variation of the Root-MeanSquared Error

EAHP $=$ Exhaust Air Heat Pump

$\mathrm{ECM}=$ Energy Conservation Measure

$\mathrm{FAO}=$ Food and Agriculture Organization

FDD $=$ Fault Detection and Diagnosis

FEMP $=$ The International Performance Measurement and Verification Protocol

GHG = Green House Gas

$\mathrm{ICE}=$ Indoor Climate and Energy

IoT = Internet of Things

IPMVP $=$ The International Performance Measurement and Verification Protocol

MAPE $=$ Mean Absolute Percentage Error

NMBE $=$ Normalized Mean Bias Error

$\mathrm{RH}=$ Relative Humidity

SA $=$ Sensitivity Analysis

$\mathrm{UA}=$ Uncertainty Analysis 